Scientific Paper

\title{
Coherence and phase synchrony analyses of EEG signals in Mild Cognitive Impairment (MCI): A study of functional brain connectivity
}

\author{
Nita HANDAYANI ${ }^{1,2, a}$, Freddy HARYANTO ${ }^{1}$, Siti Nurul KHOTIMAH ${ }^{1}$, Idam ARIF ${ }^{1}$, Warsito Purwo TARUNO ${ }^{3}$

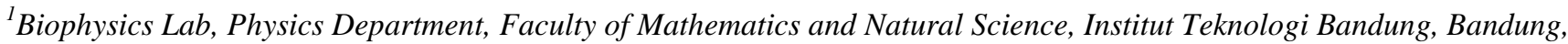 \\ Indonesia \\ ${ }^{2}$ Physics Department, Faculty of Science and Technology, UIN Sunan Kalijaga, Yogyakarta, Indonesia \\ ${ }^{3}$ Neuroscience Divison, CTech Labs, PT Edwar Technology, Tangerang, Indonesia \\ ${ }^{a}$ E-mail address: nitahandayani@yahoo.com
}

(received 5 May 2017; revised 17 August 2017; accepted 28 November 2017)

\begin{abstract}
This paper presents an EEG study for coherence and phase synchrony in mild cognitive impairment (MCI) subjects. MCI is characterized by cognitive decline, which is an early stage of Alzheimer's disease (AD). AD is a neurodegenerative disorder with symptoms such as memory loss and cognitive impairment. EEG coherence is a statistical measure of correlation between signals from electrodes spatially separated on the scalp. The magnitude of phase synchrony is expressed in the phase locking value (PLV), a statistical measure of neuronal connectivity in the human brain. Brain signals were recorded using an Emotiv Epoc 14-channel wireless EEG at a sampling frequency of $128 \mathrm{~Hz}$. In this study, we used 22 elderly subjects consisted of $10 \mathrm{MCI}$ subjects and 12 healthy subjects as control group. The coherence between each electrode pair was measured for all frequency bands (delta, theta, alpha and beta). In the MCI subjects, the value of coherence and phase synchrony was generally lower than in the healthy subjects especially in the beta frequency. A decline of intrahemisphere coherence in the MCI subjects occurred in the left temporo-parietal-occipital region. The pattern of decline in MCI coherence is associated with decreased cholinergic connectivity along the path that connects the temporal, occipital, and parietal areas of the brain to the frontal area of the brain. EEG coherence and phase synchrony are able to distinguish persons who suffer AD in the early stages from healthy elderly subjects.
\end{abstract}

Key words: EEG; mild cognitive impairment; coherence; phase synchrony; Alzheimer's disease.

\section{Introduction}

Alzheimer's disease (AD) is a progressive neurodegenerative disorder characterized by impairment of cognitive functions. The progression of AD can be categorized into four different stages. The first stage is mild cognitive impairment (MCI), an intermediate stage between the expected cognitive decline of normal aging and the more serious decline of dementia. The second and third stages are mild $\mathrm{AD}$ and moderate $\mathrm{AD}$, and are characterized by increasing cognitive deficits and difficulty to perform routine tasks. The last stage is severe $\mathrm{AD}$, where the patients lose sensitivity to their environment while memory and cognitive skills continue to worsen, and they become completely dependent on caregivers for their daily activities [1]. Early diagnosis of AD is very important to prevent disease progression to the more severe stages.

Early diagnosis of $\mathrm{AD}$ is not an easy task, because the symptoms are hard to distinguish from the symptoms of other types of dementia. One major feature in $\mathrm{AD}$ is the gradual loss of connections between neurons in the brain, which can lead to damaged cell function and eventually the death of brain cells
[2]. The cell damage is caused by the accumulation of beta amyloid plaques outside of the cells and the formation of neurofibrillary tangles inside of the cells, where the extent of the damage determines the stage of development of AD [3, 4].

EEG signals analysis is a promising technique for early diagnosis of AD [5]. Several studies of EEG data analysis have been performed for the detection of neurological abnormalities in $\mathrm{AD}$ patients. EEG slowing in patients with $\mathrm{AD}$ is a phenomenon that has often been reported by researchers and is related to the progression of the disease [6]. EEG slowing is associated with an increase in delta and theta activities and a decrease in alpha and beta activities [7-10].

In addition, EEG is a non-invasive and non-radiative examination technique that can be used to study the functional connectivity of the brain by measuring signals from the cortical brain. The cortical connectivity of the brain reflects the functional level in terms of communication between different parts of the brain. AD is characterized by low levels of functional connectivity [11]. Functional connectivity is defined as the temporal correlation between the activity of different neural assemblies [12]. According to Uhlhaas and Singer [13], 
impairment in the temporal coordination of this neuronal activity contributes to cognitive impairment in patients with $A D$.

The magnitude of the functional connectivity and synchronization between the two continuous signal can be measured by the linear and nonlinear estimators, bivariate and multivariate approaches, directed and undirected measures [14]. The measure of the linear synchronization for example cross correlation and spectral coherence, whereas the nonlinear synchronization expressed in mutual information, entropy transfer, Granger causality, or the nonlinear interdependence and phase synchronization [15].

Most of the results of previous research stated that in $\mathrm{AD}$ patients has decreased coherence spectral in alpha band compared with healthy elderly [16-19]. As for the EEG coherence of delta and theta bands, several studies have shown contradictory results, ie an increase or decrease in coherence [16,19-20]. The results of the analysis of synchronization with the Synchronization likelihood (SL) reported that in $\mathrm{AD}$ and MCI subjects loss of functional connectivity in the high frequency alpha and beta bands [10,21]. Another study reported a poor SL in $\mathrm{AD}$ patients at delta, theta, alpha and beta bands [22]. SL in MCI was lower than normal elderly people and $\mathrm{SL}$ in $\mathrm{AD}$ was lower than $\mathrm{MCI}$ in the midline and right fronto-parietal electrodes [23].

In this study, the measure of synchronization used is the spectral coherence and phase synchrony, where both are in the frequency domain. Spectral coherence and phase synchrony are the bivariate method which measures the synchronization between the two time series of EEG electrode pair. A comprehensive intrahemispheric and interhemispheric coherences from all combinations of electrode pairs, and phase synchronization of neural activity from EEG data are presented. The objective of this study was to obtain additional information about brain connectivity in MCI patients and compared to healthy elderly subjects. Data acquisition process used a portable wireless EEG system is the proposed method in this study. The overall results were statistically tested to observed any significant difference between the groups of MCI patients and the healthy elderly subjects.

\section{Materials and Methods}

\section{Subjects}

The subjects consisted of $10 \mathrm{MCI}$ patients (3 males and 7 females) at the age of $(72.20 \pm 7.29)$ years. The symptom severity was assessed using Mini Mental State Examination (MMSE) [24] and Montreal Cognitive Assesment (MoCA) [25], which revealed overall scores of $(20.30 \pm 0.82)$ and $(16.60 \pm 1.95)$ respectively. The control group consisted of 12 healthy subjects ( 4 males and 8 females) with no neurological abnormalities and no personal psychiatric history. They were aged $(70.17 \pm 4.36)$ years, had a MMSE score of $(27.25 \pm 2.00)$ and a MoCA score of $(26.50 \pm 1.24)$ (see Table 1). All subjects were volunteers who gave informed consent to undergo brain recording.

\section{EEG Recording}

EEG recording was performed using Emotiv Epoc 14 electrodes (AF3, F7, F3, FC5, T7, P7, O1, O2, P8, T8, FC6, F4, F8, AF4) according to the international 10-20 system. The EEG data were recorded at a sampling rate of $128 \mathrm{~Hz}$ with a resolution of $0.51 \mu \mathrm{V}$ and a bandwidth of $0.2-45 \mathrm{~Hz}$. Noise was filtered with notch filters at $50 \mathrm{~Hz}$ and $60 \mathrm{~Hz}$. The subjects were placed in a resting condition with their eyes closed for 20 minutes during the time of recording. Artifacts such as eyeblinking and muscle activity were rejected by visual inspection.

\section{Coherence Analysis}

Coherence is a linear measure of the correlation between two signals as a function of frequency. Coherence can be interpreted as a measure of the degree of synchronization between the brain signals in specific brain areas [26,27]. Coherence is calculated from a pair of signals, $\mathrm{x}$ and $\mathrm{y}$. The magnitude is the square cross-spectrum of the two signals divided by the product of the power spectral densities (PSD) of each signal, formulated as [28]:

$C_{x y}(f)=\frac{\left|W_{x y}\right|^{2}(f)}{W_{x}(f) * W_{y}(f)}$

Eq. 1

where $W_{x y}$ is the cross-spectral density of the two signals, $\mathrm{f}$ is the frequency, $W_{x}$ is the PSD of $x$, and $W_{y}$ is the PSD of $y$.

Absolute power values in the following frequency bands: delta $(1-4 \mathrm{~Hz})$, theta $(4-7 \mathrm{~Hz})$, alpha $(7-13 \mathrm{~Hz})$ and beta $(13-30$ $\mathrm{Hz}$ ) were used. Intrahemisphere EEG coherence was calculated for all combinations of electrode pairs. Interhemisphere coherence was calculated for the seventh electrode pairs: AF3AF4, F7-F8, F3-F4, FC5-FC6, T7-T8, P7-P8 and O1-O2, as shown in Figure 1a.

Table 1. Clinical characteristics of MCI and control subjects.

\begin{tabular}{lccc}
\hline \hline & $\begin{array}{c}\text { MCI group } \\
(\mathbf{N = 1 0})\end{array}$ & $\begin{array}{c}\text { Control group } \\
(\mathbf{N = 1 2})\end{array}$ & p-value \\
\hline Age (years) & $72.20 \pm 7.29$ & $70.17 \pm 4.36$ & $0.428^{1}$ \\
Male:Female & $3: 7$ & $4: 8$ & $0.867^{2}$ \\
MMSE score & $20.30 \pm 0.82$ & $27.25 \pm 2.00$ & $0.000^{1}$ \\
MoCA score & $16.60 \pm 1.95$ & $26.50 \pm 1.24$ & $0.000^{1}$ \\
\hline \hline
\end{tabular}

${ }^{1}$ Independent-samples t-test; ${ }^{2}$ Chi-square test

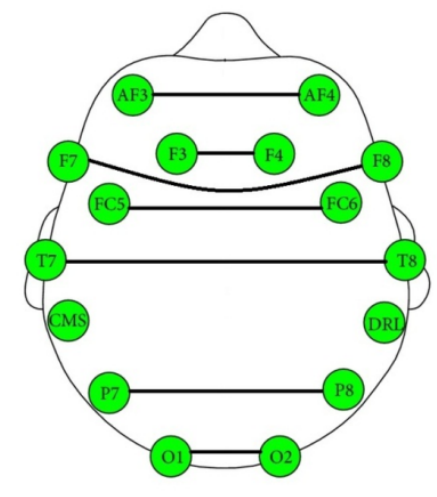

(a)

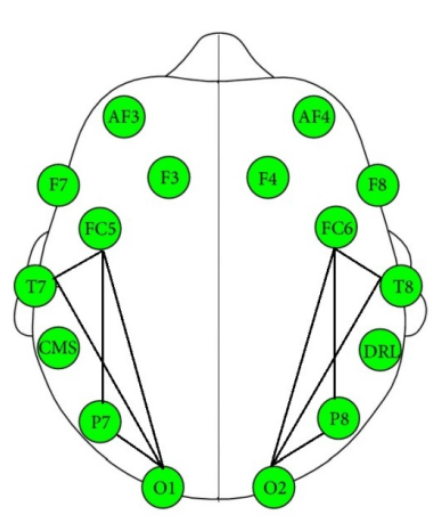

(b)
Figure 1. (a) The homologous interhemisphere electrode pairs, (b) the electrode pairs for the PLV analysis. 
For further analysis, the coherence was divided into two groups based on the distance between each pair of electrodes, i.e. local coherence and distal coherence. Local coherence for the left hemisphere included the following pairs of electrodes: AF3-F7, AF3-F3, AF3-FC5, F7-F3, F7-FC5, F7-T7, F3-FC5, FC5-T7, P7-O1. Distal coherence for the left hemisphere included the following pairs of electrodes: AF3-T7, AF3-P7, AF3-O1, F7P7, F7-O1, F3-T7, F3-P7, F3-O1, FC5-P7, FC5-O1, T7-P7, T7-O1. Local coherence in the right hemisphere included the following pairs of electrodes: AF4-F8, AF4-F4, AF4-FC6, F8F4, F8-FC6, F8-T8, F4-FC6, FC6-T8, P8-O2. Distal coherence in the right hemisphere included the following pairs of electrodes: AF4-T8, AF4-P8, AF4-O2, F8-P8, F8-O2, F4-T8, F4-P8, F4-O2, FC6-P8, FC6-O2, T8-P8, T8-O2.

\section{Phase Locking Value (PLV)}

The PLV method is a representative method for obtaining a statistical measure of the strength of phase synchronization of neural activity from EEG data. Phase synchrony refers to the interdependence between the instantaneous phases of two signals. The instantaneous phases are synchronized even if the amplitudes of both signals are statistically independent. Phase synchrony, defined as the locking of the phases associated to each signal, is expressed as [12]:

$\left|\phi_{x}(t)-\phi_{y}(t)\right|=$ constant

Eq. 2

To estimate the instantaneous phase of a signal, the Hilbert transform was used:

$H(t)=x(t)+i \tilde{x}(t)$

where $\tilde{x}(t)$ is the Hilbert transform of $x(t)$, defined as:

$\tilde{x}(t)=\frac{1}{\pi} P V \int_{-\infty}^{\infty} \frac{x\left(t^{\prime}\right)}{t-t^{\prime}} d t^{\prime}$

Eq. 4

where PV denotes the Cauchy principal value. The analytical signal phase is defined as:

$\phi(t)=\arctan \frac{\tilde{x}(t)}{x(t)}$

Eq. 5

The PLV bivariate metric for two signals, $x(t)$ and $y(t)$, is then defined as the average value:

$P L V=\left|\frac{1}{N} \sum_{j=0}^{N=1} e^{i\left(\phi_{X}(j \Delta t)-\phi_{Y}(j \Delta t)\right)}\right|$

Eq. 6

Where $N$ is the sample number of each signal and $\Delta t$ is the sampling period. The PLV takes a value within $[0,1]$, where 0 indicates a lack of phase synchronization and 1 indicates perfect phase synchronization (the phase difference varies a small amount accross the signals). PLV calculation is based on the method introduced by Lachaux [29]. The stages of PLV calculation include: (1) filtering the signal with an finite impulse response (FIR) band-pass filter, (2) calculating the instantaneous phase $(\Delta \phi)$ with the Hilbert transform, and (3) normalization of the PLV so it has a value between 0 and 1 .
The PLV analysis was performed only in areas associated with the long cortico-cortical connection which included 5 electrode pairs for the left hemisphere (FC5-T7, FC5-P7, FC5-O1, T7O1, P7-O1) and 5 electrode pairs for the right hemisphere (FC6-T8, FC6-P8, FC6-O2, T8-O2, P8-O2) as shown in Figure $1 b$.

\section{Statistical Analysis}

SPSS for Windows was used for statistical data analysis. Group differences between the patients with MCI and the healthy elderly subjects in age, MMSE and MoCA score were assessed using Independent t-test. We investigated whether there were statistically significant differences in EEG coherence between the MCI patients and the control subjects. For this purpose, we applied the Mann-Whitney $U$ test to the both of the EEG coherences (intrahemispheric and interhemispheric coherences) from the MCI patients and the healthy elderly subjects. The Mann-Whitney U test is a non-parametric test that is useful for determining if the means of two groups differ from each other. A lower $p$-value indicates a higher significance of the difference between the medians of two populations [15].

\section{Results}

Table 1 shows the results of the statistical data analysis for the MCI patients and the control subjects. The scores in the MMSE and MoCA test differed significantly between both groups, where the MCI group scored lower than the control group $(\mathrm{p}<0.05)$.

The results of the interhemisphere coherence statistical test are presented in Table 2. There is a significant difference of coherence in the electrode pair F7-F8 ( $p=0.004)$; FC5-FC6 ( $p$ $=0.038)$ for the delta band and a pair of electrodes FC5-FC6 (p $=0.003)$; T7-T8 $(p=0.009)$ for the theta band. As for the higher frequency band, there is a significant difference of coherence in the electrode pair T7-T8 ( $\mathrm{p}=0.000)$; P7-P8 ( $\mathrm{p}=$ 0.029 ) for the alpha band and a pair of electrodes AF3-AF4 (p $=0.002)$; F7-F8 ( $\mathrm{p}=0.015)$; FC5-FC6 ( $\mathrm{p}=0.008)$; P7-P8 ( $\mathrm{p}=$ 0.012 ) for the beta band. The total spectral coherence for the all combination of electrode pair showed delta coherence in the MCI group greater than the control group. Instead alpha and beta coherences in the MCI group decreased compared with the control group. For the total theta coherence, there is no significant changes.

The maximum interhemisphere coherence was observed at the electrode pair AF3-AF4 (anterior frontal area) for all frequency bands. In general, the coherence in pairs of adjacent electrodes (local coherence) showed higher values. Conversely, the coherence was reduced as a function of the spatial distance between pairs of electrodes (distal coherence) [30]. The difference in value of the interhemisphere coherence is graphically represented in Figure 2. 
Table 2. The mean value \pm standard deviation of the interhemisphere coherence for all frequency bands.

\begin{tabular}{|c|c|c|c|c|c|c|}
\hline \multirow[b]{2}{*}{ Electrode Pairs } & \multicolumn{3}{|c|}{ Delta Coherence } & \multicolumn{3}{|c|}{ Theta Coherence } \\
\hline & $\begin{array}{c}\text { MCI } \\
(\text { Mean } \pm \text { SD })\end{array}$ & $\begin{array}{c}\text { Control } \\
(\text { Mean } \pm \text { SD })\end{array}$ & p-value & $\begin{array}{c}\text { MCI } \\
(\text { Mean } \pm \text { SD })\end{array}$ & $\begin{array}{c}\text { Control } \\
(\text { Mean } \pm \text { SD })\end{array}$ & p-value \\
\hline AF3-AF4 & $0.59 \pm 0.08$ & $0.57 \pm 0.14$ & 0.741 & $0.65 \pm 0.14$ & $0.73 \pm 0.08$ & 0.198 \\
\hline F7-F8 & $0.33 \pm 0.05$ & $0.24 \pm 0.05$ & $0.004 *$ & $0.38 \pm 0.08$ & $0.36 \pm 0.12$ & 0.262 \\
\hline F3-F4 & $0.44 \pm 0.09$ & $0.38 \pm 0.17$ & 0.947 & $0.44 \pm 0.12$ & $0.43 \pm 0.15$ & 0.792 \\
\hline FC5-FC6 & $0.37 \pm 0.07$ & $0.34 \pm 0.05$ & $0.038 *$ & $0.29 \pm 0.06$ & $0.39 \pm 0.09$ & $0.003 *$ \\
\hline $\mathrm{T} 7-\mathrm{T} 8$ & $0.27 \pm 0.05$ & $0.24 \pm 0.09$ & 0.075 & $0.27 \pm 0.07$ & $0.20 \pm 0.03$ & $0.009 *$ \\
\hline P7-P8 & $0.31 \pm 0.02$ & $0.32 \pm 0.09$ & 0.468 & $0.27 \pm 0.06$ & $0.32 \pm 0.07$ & 0.166 \\
\hline $\mathrm{O} 1-\mathrm{O} 2$ & $0.47 \pm 0.06$ & $0.28 \pm 0.05$ & 0.069 & $0.32 \pm 0.13$ & $0.35 \pm 0.08$ & 0.235 \\
\hline \multirow[b]{2}{*}{ Electrode Pairs } & \multicolumn{3}{|c|}{ Alpha Coherence } & \multicolumn{3}{|c|}{ Beta Coherence } \\
\hline & $\begin{array}{c}\text { MCI } \\
(\text { Mean } \pm \text { SD }) \\
\end{array}$ & $\begin{array}{c}\text { Control } \\
(\text { Mean } \pm \text { SD })\end{array}$ & p-value & $\begin{array}{c}\text { MCI } \\
(\text { Mean } \pm \text { SD })\end{array}$ & $\begin{array}{c}\text { Control } \\
(\text { Mean } \pm \text { SD }) \\
\end{array}$ & p-value \\
\hline AF3-AF4 & $0.75 \pm 0.12$ & $0.82 \pm 0.11$ & 0.210 & $0.57 \pm 0.12$ & $0.74 \pm 0.04$ & $0.002^{*}$ \\
\hline F7-F8 & $0.44 \pm 0.08$ & $0.52 \pm 0.16$ & 0.322 & $0.40 \pm 0.11$ & $0.52 \pm 0.07$ & $0.015^{*}$ \\
\hline F3-F4 & $0.64 \pm 0.17$ & $0.58 \pm 0.15$ & 0.429 & $0.52 \pm 0.13$ & $0.63 \pm 0.08$ & 0.056 \\
\hline FC5-FC6 & $0.43 \pm 0.09$ & $0.50 \pm 0.12$ & 0.235 & $0.29 \pm 0.10$ & $0.41 \pm 0.06$ & $0.008 *$ \\
\hline T7-T8 & $0.31 \pm 0.05$ & $0.38 \pm 0.04$ & $0.000 *$ & $0.27 \pm 0.11$ & $0.32 \pm 0.08$ & 0.099 \\
\hline P7-P8 & $0.26 \pm 0.05$ & $0.30 \pm 0.03$ & $0.029 *$ & $0.22 \pm 0.05$ & $0.29 \pm 0.05$ & $0.012 *$ \\
\hline $\mathrm{O} 1-\mathrm{O} 2$ & $0.33 \pm 0.07$ & $0.40 \pm 0.08$ & 0.099 & $0.37 \pm 0.09$ & $0.43 \pm 0.07$ & 0.146 \\
\hline
\end{tabular}

*indicates $\mathrm{p}<0.05$ (Mann-Whitney U test)

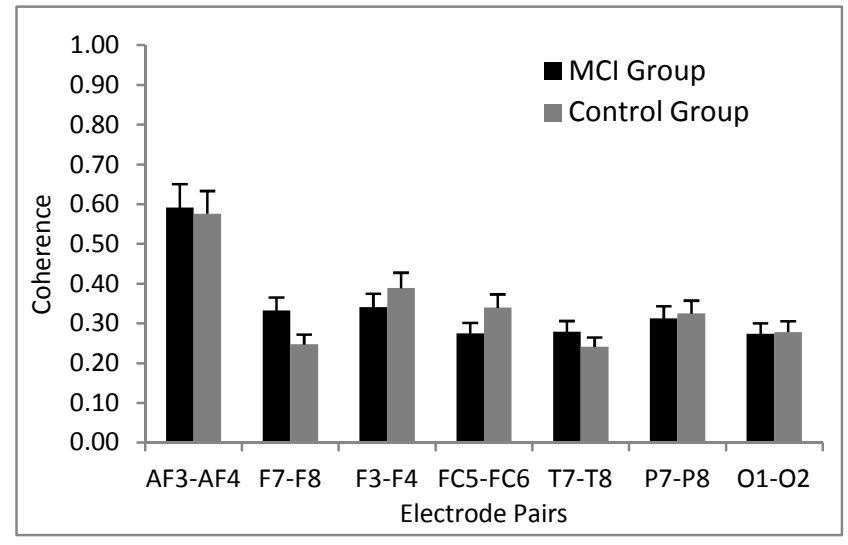

(a)

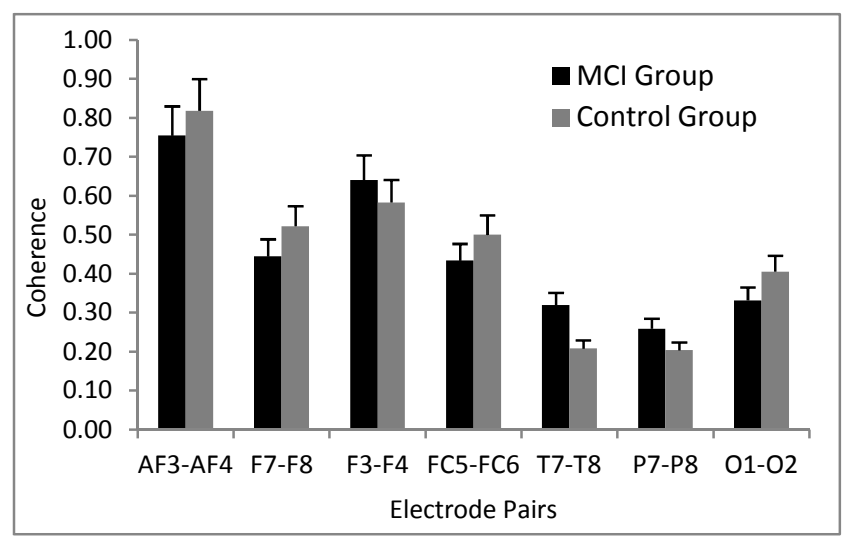

(c)

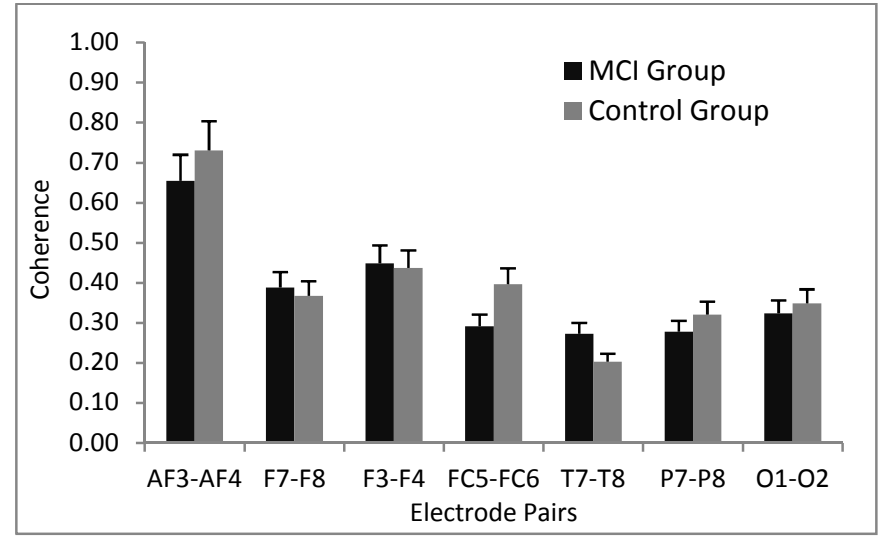

(b)

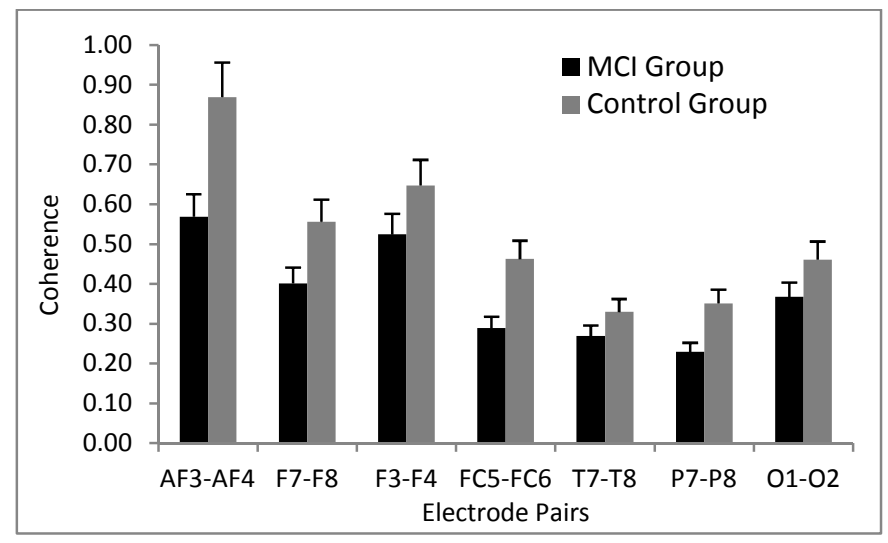

(d)

Figure 2. Comparison of interhemisphere coherence between the MCI group and the control group for delta (b) theta (c) alpha and (d) beta frequency bands. 
Coherence of electrode pairs with a statistical significance of $\mathrm{p}$ $<0.05$ at the left intrahemisphere and the right intrahemisphere shown in Table 3. From the table it appears that the left intrahemisphere has a number of pairs electrode with coherence significant difference more than the right intrahemisphere. Based on the calculation of total coherence obtained the information that coherence at the MCI group lower than the control group for the high frequency band (alpha and beta bands).

Table 4 shows the electrode pairs with maximum coherence for all frequency bands, in MCI and control groups. For the MCI group, the maximum local coherence in the delta band was observed at the electrode pair F7-FC5 (left frontal area). On the theta and alpha bands, the maximum local coherence was observed at the electrode pair F8-FC6 (right frontal area) and on the beta band it was observed at the electrode pair AF3F3 (left frontal area). The maximum distal coherence on all frequency bands was observed at the electrode pair T8-P8 (right temporo-parietal area). For the control group, the maximum local coherence in the delta band was observed at the electrode pair AF3-F7 (left frontal area). On the theta, alpha and beta bands, the maximum local coherence was observed at the electrode pair F7-FC5 (left frontal area). The maximum distal coherence in the delta and theta band was observed at the electrode pair FC6-P8 (right fronto-central-parietal area). In the beta band, the maximum distal coherence was observed at the electrode pair T8-P8 (right temporo-parietal area).
Furthermore, we observe the measure of phase synchrony in EEG data. Phase synchrony measures used was PLV. PLV statistics can be regarded as a proxy for brain connectivity. If the EEG signals from two channels under experimental conditions, exceed their baseline value simultaneously up and down, then there is synchronization (enhanced connectivity) between the two signals. If they are lower than their baseline value then there is desynchronization (decreased connectivity) between the two signals.

Table 3. Electrode pairs of the left intrahemisphere and the right intrahemisphere coherences with statistical significance of $p<$ 0.05.

\begin{tabular}{cccccc}
\hline \hline \multirow{2}{*}{$\begin{array}{c}\text { Frequency } \\
\text { Band }\end{array}$} & \multicolumn{2}{c}{ The left intrahemisphere } & & \multicolumn{2}{c}{ The right intrahemisphere } \\
\cline { 2 - 3 } \cline { 5 - 6 } Delta & Electrode Pairs & p-value & & Electrode Pairs & p-value \\
\hline \multirow{3}{*}{ Theta } & AF3-FC5 & 0.018 & & AF4-T8 & 0.041 \\
& F7-T7 & 0.048 & & FC6-O2 & 0.035 \\
\hline \multirow{5}{*}{ Alpha } & F7-FC5 & 0.021 & & \\
& F7-P7 & 0.009 & & AF4-O2 & 0.006 \\
& F3-O1 & 0.048 & & F8-O2 & 0.035 \\
& P7-O1 & 0.000 & & \\
\hline \multirow{5}{*}{ Beta } & F7-FC5 & 0.017 & & \\
& F7-O1 & 0.021 & & AF4-O2 & 0.044 \\
& FC5-P7 & 0.017 & & F8-O2 & 0.003 \\
& FC5-O1 & 0.018 & & \\
& P7-O1 & 0.015 & & \\
\hline \hline & AF3-P7 & 0.002 & & \\
\hline & F7-FC5 & 0.010 & & AF4-F4 & 0.023 \\
& F7-O1 & 0.025 & F8-O2 & 0.041 \\
& FC5-O1 & 0.019 & & \\
\hline
\end{tabular}

Table 4. Electrode pairs with maximum intrahemisphere coherence for all frequency bands.

\begin{tabular}{|c|c|c|c|c|c|c|c|c|}
\hline \multirow{2}{*}{ Electrode } & \multicolumn{2}{|c|}{ Delta } & \multicolumn{2}{|c|}{ Theta } & \multicolumn{2}{|c|}{ Alpha } & \multicolumn{2}{|c|}{ Beta } \\
\hline & MCI & Control & MCI & Control & MCI & Control & MCI & Control \\
\hline \multirow{2}{*}{ AF3 } & F3 & F7 & F3 & F3 & F3 & F3 & F3 & F3 \\
\hline & $(0.488)$ & $(0.547)^{*}$ & $(0.576)$ & $(0.671)$ & $(0.734)$ & $(0.769)$ & $(0.618)^{*}$ & $(0.632)$ \\
\hline \multirow{2}{*}{ F7 } & FC5 & AF3 & FC5 & FC5 & FC5 & FC5 & FC5 & FC5 \\
\hline & $(0.502)^{*}$ & $(0.547)^{*}$ & $(0.576)$ & $(0.722)^{*}$ & $(0.646)$ & $(0.846)^{*}$ & $(0.458)$ & $(0.688)^{*}$ \\
\hline \multirow{2}{*}{$\mathrm{F} 3$} & AF3 & AF3 & AF3 & AF3 & AF3 & AF3 & AF3 & AF3 \\
\hline & $(0.488)$ & $(0.518)$ & $(0.576)$ & $(0.671)$ & $(0.734)$ & $(0.796)$ & $(0.618)^{*}$ & $(0.632)$ \\
\hline \multirow{2}{*}{ FC5 } & F7 & F7 & F7 & F7 & F7 & F7 & F3 & F7 \\
\hline & $(0.502)^{*}$ & $(0.536)$ & $(0.576)$ & $(0.722)^{*}$ & $(0.646)$ & $(0.846)^{*}$ & $(0.463)$ & $(0.688)^{*}$ \\
\hline \multirow{2}{*}{$\mathrm{T} 7$} & FC5 & F7 & FC5 & F7 & F7 & F7 & P7 & F7 \\
\hline & $(0.435)$ & $(0.441)$ & $(0.426)$ & $(0.393)$ & (0.399) & $(0.366)$ & $(0.308)$ & $(0.368)$ \\
\hline \multirow{2}{*}{ P7 } & $\mathrm{T} 7$ & $\mathrm{~T} 7$ & $\mathrm{~T} 7$ & $\mathrm{O} 1$ & $\mathrm{O} 1$ & $\mathrm{O} 1$ & $\mathrm{O} 1$ & $\mathrm{O} 1$ \\
\hline & $(0.381)$ & $(0.339)$ & $(0.345)$ & $(0.557)$ & $(0.375)$ & $(0.504)$ & $(0.389)$ & $(0.483)$ \\
\hline \multirow{2}{*}{ O1 } & P7 & P7 & $\mathrm{P} 7$ & P7 & $\mathrm{P} 7$ & $\mathrm{P} 7$ & P7 & P7 \\
\hline & $(0.363)$ & $(0.424)$ & $(0.301)$ & $(0.557)$ & $(0.375)$ & $(0.504)$ & $(0.389)$ & $(0.483)$ \\
\hline \multirow{2}{*}{$\mathrm{O} 2$} & P8 & P8 & P8 & P8 & P8 & P8 & P8 & P8 \\
\hline & $(0.427)$ & $(0.385)$ & $(0.412)$ & $(0.500)$ & $(0.475)$ & $(0.580)$ & $(0.488)$ & $(0.511)$ \\
\hline \multirow{2}{*}{ P8 } & $\mathrm{T} 8$ & FC6 & T8 & FC6 & $\mathrm{O} 2$ & $\mathrm{O} 2$ & $\mathrm{O} 2$ & $\mathrm{~T} 8$ \\
\hline & $(0.482)$ & $(0.436)$ & $(0.484)$ & $(0.532)$ & $(0.475)$ & $(0.580)$ & $(0.488)$ & $(0.545)$ \\
\hline \multirow{2}{*}{$\mathrm{T} 8$} & P8 & FC6 & P8 & P8 & P8 & FC6 & P8 & P8 \\
\hline & $(0.482)$ & $(0.363)$ & $(0.484)$ & $(0.470)$ & $(0.629)$ & $(0.557)$ & $(0.483)$ & $(0.545)$ \\
\hline \multirow{2}{*}{ FC6 } & F8 & $\mathrm{AF} 4$ & F8 & $\mathrm{F} 8$ & F8 & F8 & $\mathrm{F} 4$ & F8 \\
\hline & $(0.425)$ & $(0.519)$ & $(0.646)^{*}$ & $(0.718)$ & $(0.747)^{*}$ & (0.829) & $(0.501)$ & $(0.686)$ \\
\hline \multirow{2}{*}{$\mathrm{F} 4$} & $\mathrm{~T} 8$ & AF4 & $\mathrm{AF} 4$ & AF4 & AF4 & AF4 & AF4 & AF4 \\
\hline & $(0.330)$ & $(0.403)$ & $(0.511)$ & $(0.498)$ & $(0.703)$ & (0.639) & $(0.592)$ & $(0.480)$ \\
\hline \multirow{2}{*}{ F8 } & AF4 & $\mathrm{AF} 4$ & FC6 & FC6 & FC6 & FC6 & AF4 & FC6 \\
\hline & $(0.476)$ & $(0.419)$ & $(0.646)^{*}$ & $(0.718)$ & $(0.747)^{*}$ & $(0.829)$ & $(0.529)$ & $(0.686)$ \\
\hline \multirow{2}{*}{$\mathrm{AF} 4$} & F8 & FC6 & F8 & F8 & $\mathrm{F} 4$ & F8 & $\mathrm{F} 4$ & F8 \\
\hline & $(0.476)$ & (0.519) & $(0.573)$ & $(0.626)$ & $(0.703)$ & $(0.735)$ & $(0.592)$ & $(0.542)$ \\
\hline
\end{tabular}


Table 5. The mean value \pm standard deviation of PLV in MCI group and control group for alpha band.

\begin{tabular}{cccc}
\hline \hline Electrode Pairs & $\begin{array}{c}\text { MCI } \\
(\text { Mean } \pm \text { SD })\end{array}$ & $\begin{array}{c}\text { Control } \\
(\text { Mean } \pm \text { SD })\end{array}$ & p-value \\
\hline FC5-T7 & $0.1464 \pm 0.0117$ & $0.1482 \pm 0.0045$ & 0.151 \\
FC5-P7 & $0.1444 \pm 0.0085$ & $0.1497 \pm 0.0089$ & $0.044^{*}$ \\
FC5-O1 & $0.1480 \pm 0.0074$ & $0.1468 \pm 0.0067$ & 0.734 \\
T7-O1 & $0.1444 \pm 0.0053$ & $0.1514 \pm 0.0072$ & $0.014^{*}$ \\
P7-O1 & $0.1468 \pm 0.0041$ & $0.1480 \pm 0.0046$ & 0.650 \\
FC6-T8 & $0.1445 \pm 0.0072$ & $0.1454 \pm 0.0067$ & 0.740 \\
FC6-P8 & $0.1484 \pm 0.0071$ & $0.1495 \pm 0.0064$ & 0.545 \\
FC6-O2 & $0.1464 \pm 0.0082$ & $0.1432 \pm 0.0081$ & 0.326 \\
T8-O2 & $0.1491 \pm 0.0074$ & $0.1451 \pm 0.0050$ & 0.053 \\
P8-O2 & $0.1449 \pm 0.0058$ & $0.1492 \pm 0.0035$ & $0.049 *$ \\
\hline \hline
\end{tabular}

*indicates $\mathrm{p}<0.05$ (Mann-Whitney U test)

Table 6. The mean value \pm standard deviation of PLV in MCI group and control group for beta band.

\begin{tabular}{cccc}
\hline \hline Electrode Pairs & $\begin{array}{c}\text { MCI } \\
(\text { Mean } \pm \text { SD })\end{array}$ & $\begin{array}{c}\text { Control } \\
(\text { Mean } \pm \text { SD })\end{array}$ & p-value \\
\hline FC5-T7 & $0.1401 \pm 0.0027$ & $0.1484 \pm 0.0037$ & $0.039^{*}$ \\
FC5-P7 & $0.1360 \pm 0.0060$ & $0.1430 \pm 0.0045$ & $0.047^{*}$ \\
FC5-O1 & $0.1384 \pm 0.0042$ & $0.1441 \pm 0.0040$ & $0.045^{*}$ \\
T7-O1 & $0.1404 \pm 0.0067$ & $0.1433 \pm 0.0050$ & 0.273 \\
P7-O1 & $0.1382 \pm 0.0048$ & $0.1477 \pm 0.0041$ & $0.026^{*}$ \\
FC6-T8 & $0.1380 \pm 0.0050$ & $0.1440 \pm 0.0035$ & 0.087 \\
FC6-P8 & $0.1390 \pm 0.0050$ & $0.1506 \pm 0.0065$ & $0.012^{*}$ \\
FC6-O2 & $0.1429 \pm 0.0038$ & $0.1435 \pm 0.0030$ & 0.344 \\
T8-O2 & $0.1411 \pm 0.0047$ & $0.1475 \pm 0.0044$ & $0.042^{*}$ \\
P8-O2 & $0.1392 \pm 0.0052$ & $0.1422 \pm 0.0030$ & 0.199 \\
\hline \hline
\end{tabular}

*indicates $\mathrm{p}<0.05$ (Mann-Whitney U test)

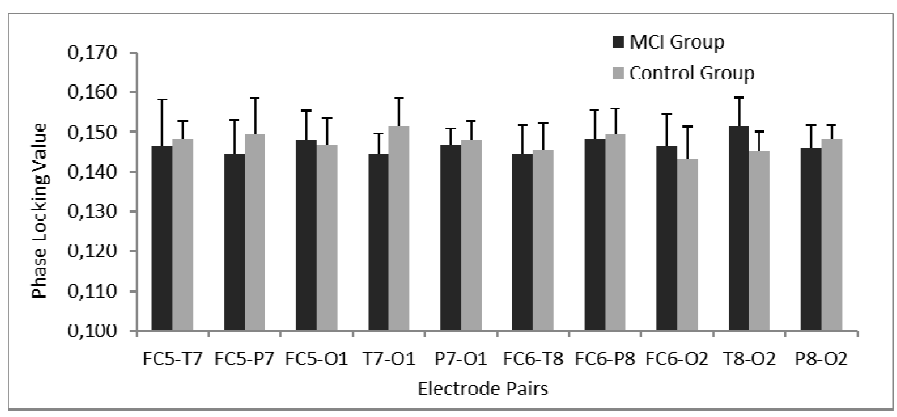

Figure 4. Comparison of phase locking value between the MCI group and the control group for the long cortico-cortical connection in the alpha frequency.

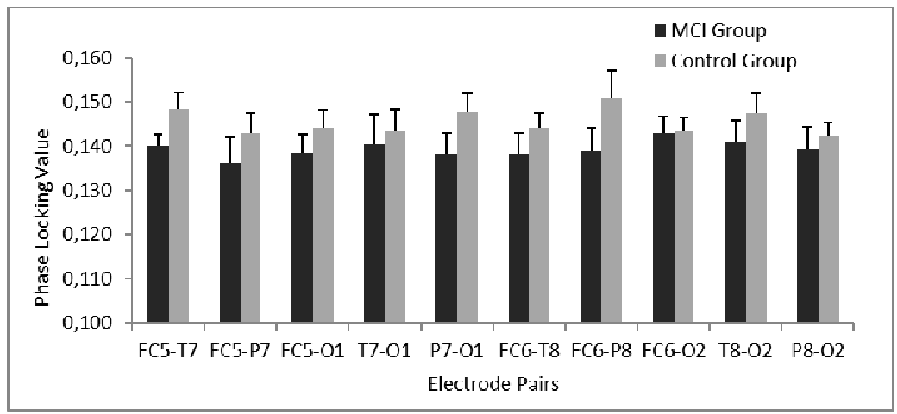

Figure 5. Comparison of phase locking value between the MCI group and the control group for the long cortico-cortical connection in the beta frequency.
In this study, the PLV calculation focused on areas related to the long cortico-cortical connection, i.e. the connection between the area of temporo-parieto-occipital to the frontal area, both in the left and the right hemisphere. The results of PLV calculation on the MCI group and the control group was only performed for the alpha and beta frequencies (Tables 5 and 6). Comparisons of the PLVs from the MCI group and the control group are respectively presented in Figures $\mathbf{4}$ and $\mathbf{5}$. From Figure 4 shows that in the MCI group, there are seven pairs of electrodes that have PLV lower than the control group at the alpha band. The seven electrode pairs are the left frontocentral-temporal (FC5-T7), the left frontocentral-parietal (FC5-P7), the left temporo-occipital (T7- O1), the left parietooccipital (P7-O1), the right frontocentral-temporal (FC6-T8), the right frontocentral-parietal (FC6-P8) and the right parietooccipital (P8-O2). In the beta band, it appears that all of the PLVs in the MCI group were lower when compared to the control group.

\section{Discussion}

The interhemispheric coherence in elderly subjects in a resting state decreases with advancing age [31]. In the present research, the interhemispheric coherence of the MCI group for the delta and theta bands increased on several pairs of electrodes. In the frontal and temporal areas, the interhemispheric coherence in the MCI group was greater than in the control group. Meanwhile, the interhemispheric coherence in the alpha band decreased at almost all pairs of electrodes. The decrease in interhemispheric coherence in the occipital area is consistent with results that have been reported by Anghinah [32]. For the beta band, the interhemispheric coherence in the control group was higher than in the MCI group for all electrode pairs.

There was an increase in the left intrahemispheric temporoparietal coherence and a decrease in the right intrahemispheric temporo-parietal coherence in the MCI group compared to the control group for almost all bands, with the exception of the beta band. These findings are consistent with the result that was reported by Sankari [28]. In neurology it is well-known that in the early stages of AD changes occur in the left temporo-parietal area, followed by changes in the right temporo-parietal area.

The increase in delta coherence could be related to a lack of influence of subcortical cholinergic structures on cortical electrical activity [16]. In patients with $\mathrm{AD}$, further reductions in the interhemispheric alpha band $(8-12 \mathrm{~Hz})$, in the coherence between occipital lobes [32] and in the temporo-parietooccipital areas [16] have been reported. An increase in delta (0$3 \mathrm{~Hz}$ ) coherence between the frontal and the posterior areas and the decrease in alpha coherence could be related to alterations in cortico-cortical connections. Leutcher [17] have stated that the decrease in alpha coherence is due to the impairment of cortico-cortical connections, especially from long fibers that connect the temporo-parieto-occipital to the frontal area of the 
brain. The earliest spectral changes in AD patients at resting state are an increase in theta activity accompanied by a decrease in beta activity, which are followed by a decrease in alpha activity [33]. The delta frequency increases later during the course of the disease [34-35].

Several medical studies have reported that some types of neurophysiological disease, including $\mathrm{AD}$, are associated with abnormalities of neural synchrony. The brain signals in different areas become less correlated in patients with $\mathrm{AD}$ [36]. Several studies have reported a decrease in the magnitude and the phase coherence of the EEGs of patients with MCI and AD [16,32,36-39]. However, other studies have reported that no effects on phase coherence were observed between $\mathrm{AD}$ and the control group [21] or between MCI and the control group [40].

Phase synchronization is the quantity that describes the measure of the functional connectivity and neural activity of the brain based on EEG signals. In this research, the measure of the signal synchronization was expressed in the PLV. The PLV calculation method, based on the Hilbert transformation, was developed by Lachaux [29]. Based on a comparison of the PLVs in the alpha band, it was shown that the PLVs for the right temporo-occipital area and both the left and the right frontocentral-occipital area were higher in the MCI group than in the control group. Meanwhile, for the beta band, the PLVs of the MCI group were lower than those of the control group for all pairs of electrodes. Therefore, it can be said that the MCI patients experienced a loss of beta-band synchronization.

Stam [21] have reported that $\mathrm{AD}$ patients experience loss of beta-band synchronization, which is correlated with more severe cognitive dysfunction. This condition is reflected in a lower MMSE score. Some researchers have examined the importance of synchronization at high frequencies for cognitive processes [41]. Loss of beta-band synchronization does not occur simply because of aging, but rather indicates the specific pathology of AD.

In the present work, bivariate estimators are used for brain functional connectivity studies of EEG data. According to Kaminski and Blinowska (2014) this type of estimator has disadvantages associated with common feeding of many spurious connections $[\mathrm{N}(\mathrm{N}-1) / 2-\mathrm{N}]$ so that it exceeds the actual number of connections [42]. This problem can actually be solved by using a multivariate estimator. But the multivariate approach is less sensitive than bivariate estimators associated with false indirect connections [43]. The choice of methods for measuring brain connectivity depends on several aspects such as robustness of inputs, noise and volume conduction [44]. Phase synchrony, mostly the PLV is one of the most commonly used methods of neural coupling [45].

Despite the lack of common feeding related spurious connections, the results of this study found statistically significant differences in coherence and synchronization values between MCI and control groups. This difference is observed in some electrode pairs for specific brain wave frequencies. The findings related to the loss of beta band synchronization mentioned above may be used for early diagnosis of AD.

\section{Conclusions}

This paper presented coherence of electrode pairs as observed in four frequency bands and phase synchrony in the alpha and beta bands. This reported research confirms the results of previous coherence studies on MCI patients that showed a decrease in both the intrahemispheric and the interhemispheric coherence especially in the beta band. Based on the measurement of phase synchrony in patients with MCI, a decrease in signal synchronization occurs in some electrode pairs for the alpha band and on all electrode pairs for beta band. Electrophysiological measures such as coherence and phase synchrony may provide an alternative screening method for early detection of MCI in potential AD patients. In the next research, will be studied in more detail about EEG data analysis using multivariate method.

\section{Acknowledgments}

The author would like to thank all volunteers for their participation in this study, especially the elderly from the Nursing Home Foundation Bandung, Indonesia. This work was partially supported by a grant from PUPT Ristekdikti 2016 (No. 584o/I1.C01/PL /2016).

\section{References}

[1] Dauwels J, Vialatte F, Latchoumane C, et al. EEG synchrony analysis for early diagnosis of Alzheimer's disease: A study with several synchrony measures and EEG datasets. Conf Proc IEEE Eng Med Biol Soc. 2009;2009:2224-2227.

[2] Petrella JR, Coleman RE, Doraiswamy PM. Neuroimaging and early diagnosis of Alzheimer disease: a look to the future. Radiology. 2003;226(2):315-36.

[3] Braak H, Braak E. Staging of Alzheimer's disease-related neurofibrillary changes. Neurobiol Aging. 1995;16(3):271-278.

[4] Czigler B, Csikós D, Hidasi Z, et al. Quantitative EEG in early Alzheimer's disease patients-power spectrum and complexity features. Int J Psychophysiol. 2008;68(1):75-80.

[5] Adeli H, Ghosh-Dastidar S, Dadmehr N. A spatio-temporal wavelet-chaos methodology for EEG-based diagnosis of Alzheimer's disease. Neurosci Lett. 2008;444(2):190-194. 
[6] Coben LA, Danziger WL, Storandt M. A longitudinal EEG study of mild senile dementia of Alzheimer type: changes at 1 year and at 2.5 years. Electroencephalogr Clin Neurophysiol. 1985;61(2):101-112.

[7] Giaquinto S, Nolfe G. The EEG in the normal elderly: a contribution to the interpretation of aging and dementia. Electroencephalogr Clin Neurophysiol. 1986;63(6):540-546.

[8] Brenner RP, Ulrich RF, Spiker DG, et al. Computerized EEG spectral analysis in elderly normal demented and depressed subjects. Electroencephalogr Clin Neurophysiol. 1986;64(6):483-492.

[9] Bennys K, Rondouin G, Vergnes C, Touchon J. Diagnostic value of quantitative EEG in Alzheimer's disease. Neurophysiol Clin. 2001;31(3):153-160.

[10] Pijnenburg YAL, v d Made Y, van Cappellen van Walsum AM, et al. EEG synchronization likelihood in mild cognitive impairment and Alzheimer's disease during a working memory task. Clin Neurophysiol. 2004;115(6):1332-1339.

[11] Stam CJ, Montez T, Jones BF, et al. Disturbed fluctuations of resting state EEG synchronization in Alzheimer's disease. Clin Neurophysiol. 2005;116(3):708-715.

[12] Sakkalis V. Review of advanced techniques for the estimation of brain connectivity measured with EEG/MEG. Comput Biol Med. 2011;41(12):1110-1117.

[13] Uhlhaas PJ, Singer W. Neural synchrony in brain disorders: relevance for cognitive dysfunctions and pathophysiology. Neuron. 2006;52(1):155-168.

[14] Blinowska KJ, Kamiński M, Brzezicka A, Kamiński J. Application of directed transfer function and network formalism for the assessment of functional connectivity in working memory task. Philos Trans A Math Phys Eng Sci. 2013;371(1997):20110614.

[15] Dauwels J, Vialatte F, Musha T, Cichocki A. A comparative study of synchrony measures for the early diagnosis of Alzheimer's disease based on EEG. NeuroImage. 2010;49(1):668-693.

[16] Locatelli T, Cursi M, Liberati D, et al. EEG coherence in Alzheimer's disease. Electroencephalogr Clin Neurophysiol. 1998;106(3):229-237.

[17] Leuchter AF, Spar JE, Walter DO, Weiner H. Electroencephalograpic spectra and coherence in the diagnosis of Alzheimer's-type and multi-infarct dementia: a pilot study. Arch Gen Psychiatry. 1987;44(11):993-998.

[18] Jelic V, Julin P, Shigeta M, et al. Apolipoprotein E $\varepsilon 4$ allele decreases functional connectivity in Alzheimer's disease as measured by EEG coherence. J Neurol Neurosurg Psychiatry. 1997;63(1):59-65.

[19] Adler G, Brassen S, Jajcevic A. EEG coherence in Alzheimer's dementia. J Neural Transm. 2003;110(9):1051-1058.

[20] Brunovsky M, Matousek M, Edman A, et al. Objective assessment of the degree of dementia by means of EEG. Neuropsychobiology. 2003;48(1):19-26.

[21] Stam CJ, van der Made Y, Pijnenburg YAL, Scheltens PH. EEG synchronization in mild cognitive impairment and Alzheimer's disease. Acta Neurol Scand. 2003 Aug;108(2):90-96.

[22] Babiloni C, Ferri R, Moretti DV, et al. Abnormal fronto-parietal coupling of brain rhythms in mild Alzheimer's disease: a multricentric EEG study. Eur J Neurosci. 2004;19(9):2583-2590.

[23] Babiloni C, Ferri R, Binetti G, et al. Fronto-parietal coupling of brain rhythms in mild cognitive impairment: a multicentric EEG study. Brain Res Bull. 2006;69(1):63-73.

[24] Folstein MF, Folstein SE, McHugh PR. "Mini-Mental State": a practical method for grading the cognitive state of patients for the clinician. J Psychiatr Res. 1975;12(3):189-198.

[25] Nasreddine ZS, Phillips NA, Bédirian V, et al. The Montreal Cognitive Assessment, MoCA: a brief screening tool for mild cognitive impairment. J Am Geriatr Soc. 2005;53(4):695-699.

[26] Nunez PL, Srinivasan R, Westdorp AF, et al. EEG coherency I: Statistics reference electrode volume conduction Laplacians cortical imaging and interpretation at multiple scales. Electroencephalogr Clin Neurophysiol. 1997;103(5):499-515.

[27] Nunez PL, Silberstein RB, Shi Z, et al. EEG coherency II: experimental comparisons of multiple measures. Clin Neurophysiol. 1999;110(3):469-486.

[28] Sankari Z, Adeli H, Adeli A. Intrahemispheric, interhemispheric, and distal EEG coherence in Alzheimer's disease. Clin Neurophysiol. 2011;122(5):897-906.

[29] Lachaux JP, Rodrigues E, Martinerie J, Varela FJ. Measuring phase synchrony in brain signals. Hum Brain Mapp. 1999;8(4):194208.

[30] Glass A, Zappulla R, Nieves J, Diamond S. EEG coherence as a predictor of spike propagation. Electroencephalogr Clin Neurophysiol. 1992;82(1):10-16.

[31] Kikuchi M, Wada Y, Koshino Y, et al. Effect of normal aging upon interhemispheric EEG coherence: analysis during rest and photic stimulation. Clin Electroencephalogr. 2000;31(4):170-174.

[32] Anghinah R, Kanda PA, Jorge MS, et al. Alpha band coherence analysis of EEG in healthy adult's and Alzheimer's type dementia patients. Arq Neuropsiquiatr. 2000;58(2A):272-275. 
[33] Jelic V, Shigeta M, Julin P, et al. Quantitative electroencephalography power and coherence in Alzheimer's disease and mild cognitive impairment. Dementia. 1996;7(6):314-323.

[34] Soininen H, Riekkinen PJ. EEG in diagnostics and follow-up of Alzheimer's disease. Acta Neurol Scand Suppl. 1992;139:36-39.

[35] Elmstahl S, Rosen I, Gullberg B. Quantitative EEG in elderly patients with Alzheimer's disease and healthy controls. Dementia. 1994;5(2):119-124.

[36] Hogan MJ, Swanwick GR, Kaiser J, et al. Memory-related EEG power and coherence reductions in mild Alzheimer's disease. Int J Psychophysiol. 2003;49(2):147-263.

[37] Jeong J. EEG dynamics in patients with Alzheimer's disease. Clin Neurophysiol. 2004;115(7):1490-1505.

[38] Besthorn C, Forstl H, Geiger-Kabish C, et al. EEG coherence in Alzheimer disease. Electroenceph Clin Neurophysiol. 1994;90(3):242-245.

[39] Güntekin B, Saatçi E, Yener G. Decrease of evoked delta, theta and alpha coherences in Alzheimer patients during a visual oddball paradigm. Brain Res. 2008;1235:109-116.

[40] Jiang ZY. Study on EEG power and coherence in patients with mild cognitive impairment during working memory task. J Zhejiang Univ Sci B. 2005;6(12):1213-1219.

[41] Basar E, Basar-Erogluc C, Karakas S, Schurmanna M. Gamma, alpha, delta, and theta oscillations govern cognitive processes. Int J Psychophysiol. 2001;39(2-3):241-248.

[42] Kaminski M, Blinowska KJ. Directed Transfer Function is not influenced by volume conduction-inexpedient pre-processing should be avoided. Front Comput Neurosci. 2014;8:61.

[43] Olejarczyk E, Marzetti L, Pizzella V, Zappasodi F. Comparison of connectivity analyses for resting state EEG data. J Neural Eng. 2017;14(3):036017.

[44] Kaminski M, Brzezicka A, Kaminski J, Blinowska K. Measures of coupling between neural populations based on Granger causality principle. Frontiers Comput Neurosci. 2016;10:114.

[45] Burgess AP. On the interpretation of synchronization in EEG hyperscanning studies: a cautionary note. Front Hum Neurosci. 2013;7:881. 